\title{
A Block Cipher Having a Key on One Side of the Plain Text Matrix and its Inverse on the Other Side
}

\author{
Dr. V. U. K. Sastry ${ }^{1}$, Prof. D. S. R. Murthy ${ }^{2}$, Dr. S. Durga Bhavani ${ }^{3}$
}

\begin{abstract}
In this paper, we have modified the Hill cipher by developing an iterative procedure consisting of three steps. In the first step, the plain text matrix is multiplied with the key matrix on one side and with its inverse on the other side. In the second step, the plain text matrix is mixed thoroughly by using a function called Mix ( ). In the last step, the plain text matrix is modified by using XOR operation between the plain text matrix and the key matrix. The cryptanalysis and the avalanche effect discussed in this paper, conspicuously indicate that the cipher is a strong one and it is quite comparable with the other block ciphers.
\end{abstract}

Index Terms-Block cipher, Key, Modular arithmetic inverse, Encryption, Decryption.

\section{INTRODUCTION}

In a recent paper [1], we have developed a block cipher by modifying the Hill cipher. In this, we have introduced an iterative scheme, which includes the key $\mathrm{K}$ as a multiplier on both the sides of the plain text matrix. Here, we have adopted a procedure, in which we have used a function called Mix () for mixing the plain text at every stage of the iteration, and applied XOR operation between the plain text matrix and the key matrix. In this analysis, we have seen that the strength of the cipher is quite significant and it cannot be broken by any cryptanalytic attack.

In the present paper, our objective is to develop another modification of the Hill cipher, by including both $\mathrm{K}_{\text {and }} \mathrm{K}^{-1}$ (one on the left side and another on the right side of the plain text matrix) in encryption as well as in decryption, instead of having only $\mathrm{K}$ in encryption and $\mathrm{K}^{-1}$ in decryption. As in [1], here also we have made use of Mix ( ) function and applied the XOR operation between the plain text matrix and the key matrix.

In section 2, we have presented the development of the cipher. We have illustrated the cipher in two different cases, and exhibited the avalanche effect in section 3 . We have discussed the cryptanalysis in section 4 . Finally, we have drawn conclusions in section 5 .

Manuscript received January 9, 2010.

${ }^{1}$ Dr. V. U. K. Sastry is with Dept. of Computer Science \& Engg., SNIST, Hyderabad, India, (e-mail: vuksastry@rediffmail.com).

${ }^{2}$ Prof. D. S. R. Murthy is with Dept. of Information Technology, SNIST, Hyderabad, India, (e-mail: dsrmurthy.1406@gmail.com).

${ }^{3}$ Dr. S. Durga Bhavani is with School of Information Technology, SIT, JNTUH, Hyderabad, India, (e-mail: sdurga.bhavani@gmail.com).

\section{DeVElopment OF THE CIPHER}

Consider a plain text $\mathrm{P}$ which can be represented in the form of a square matrix given by

$$
\mathbf{P}=\left[\mathbf{P}_{\mathrm{ij}}\right], \mathrm{i}=1 \text { to } \mathrm{n}, \mathrm{j}=1 \text { to } \mathrm{n},
$$

where each $P_{\mathrm{ij}}$ is equal to 0 or 1 .

Let us choose a key $\mathrm{k}$. Let it be represented in the form of a matrix given by

$$
\mathbf{K}=\left[\mathbf{K}_{\mathrm{ij}}\right], \mathrm{i}=1 \text { to } \mathrm{n}, \mathrm{j}=1 \text { to } \mathrm{n},
$$

where each $K_{\mathrm{ij}}$ is a binary number.

Let

$$
\mathbf{C}=\left[\mathbf{C}_{\mathrm{ij}}\right], \quad \mathrm{i}=1 \text { to } \mathrm{n}, \mathrm{j}=1 \text { to } \mathrm{n}
$$

be the corresponding cipher text matrix.

The process of encryption and the process of decryption adopted in this analysis are given in Fig. 1.

Here $r$ denotes the number of rounds in the iteration process. In the process of encryption, we have the iteration scheme which includes the relations

$$
\begin{aligned}
& \mathbf{P}=\left(\mathrm{KPK}^{-1}\right) \bmod 2, \\
& \mathbf{P}=\mathbf{M i x}(\mathbf{P}), \\
& \text { and } \mathbf{P}=\mathbf{P} \oplus \mathbf{K} .
\end{aligned}
$$

The relation (2.4) is used to achieve diffusion, while the relations (2.5) and (2.6) are used to acquire confusion. The function Mix (P) mixes the plain text at every stage of the iteration. For a detailed discussion of this function, we may refer to [1]. In the process of decryption, the function IMix represents the reverse process of Mix.
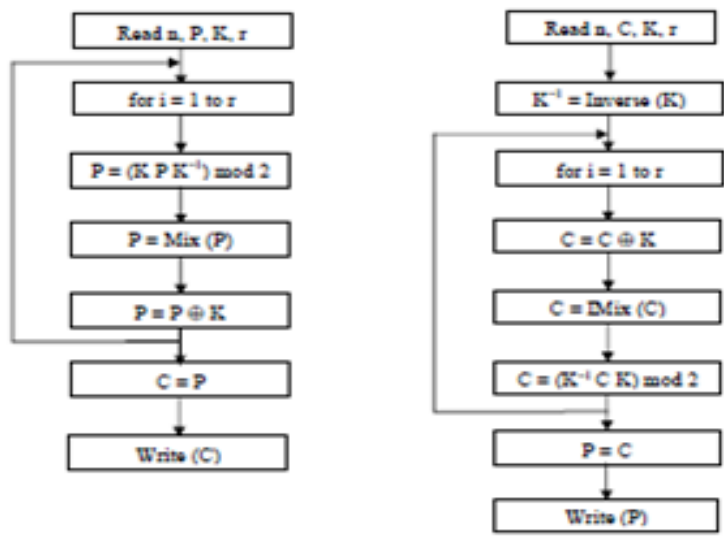

(a) Process of Encryption

(b) Process of Decryption

Fig. Scbematic diagram of the cipber

In what follows, we present the algorithms for encryption, decryption, and for the modular arithmetic inverse of a square matrix. 


\section{Algorithm for Encryption}

1. Read n, P, K, r

2. $\mathrm{K}^{-1}=$ Inverse $(\mathrm{K})$

3. for $\mathrm{i}=1$ to $\mathrm{r}$

$$
\text { f }
$$

$$
\mathrm{P}=\left(\mathrm{K} \mathrm{P} \mathrm{K}^{-1}\right) \bmod 2
$$

$\mathrm{P}=\mathrm{Mis}(\mathrm{P})$

$\mathrm{P}=\mathrm{P} \in \mathrm{K}$

\}

4. $C=P$

5. Write (C)

\section{Algorithm for Decryption}

1. Read n, C, K, r

2. $\mathrm{K}^{-1}=$ Inverse $(\mathrm{K})$

3. for $\mathrm{i}=1$ to $\mathrm{r}$

f

$$
\mathrm{C}=\mathrm{C} \in \mathrm{R}
$$$$
\mathrm{C}=\mathrm{IMix}(\mathrm{C})
$$$$
\mathrm{C}=\left(\mathrm{K}^{-1} \mathrm{C} \mathrm{K}\right) \bmod 2
$$

\}

$$
\text { 4. } \mathrm{p}=\mathrm{C}
$$

5. Write (P)

\section{Algorithm for Inverse (K)}

// The arithmetic inverse $\left(\mathrm{A}^{-5}\right)$, and the determinant of the matrix $(\Delta)$ are obtained by Gauss recuction method.

1. $A=R, N=2$

2. $A^{-1}=\left[A_{,}\right] / \Delta, i=1$ to $n, j=1$ to $n \quad / / A_{j}$ are the cofactors of $a_{i j}$, where $a_{i j}$ are elements of $A$, and $\Delta$ is the determinant of $A$

3. for $i=1$ to $\mathrm{n}$

\{

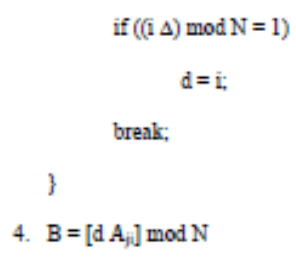

// B is the modular arithmetic inverse of A

\section{ILLUSTRATION OF THE CIPHER}

Let us consider the following plain text.

Just now we have received the news from the border security that all the planes coming from north west corner of the country by today mid-night are containing terrorists. Shoot the planes without any second thought. Finish! (3.1)

Let us focus our attention on the first 32 characters of the above plain text. This is given by

Just now we have received the ne.

On using the EBCDIC code, the plain text under consideration can be written in the Hexadecimal notation as follows:

\section{D1 A4A2 A3 40 95 96 A6 40 A6 85408881 A5 85}

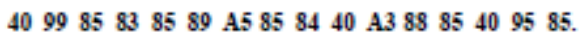

On placing two numbers in each row, that is, D1 A4 in the first row, A2 A3 in the second row, etc., the plain text matrix $\mathrm{P}$ can be written in the binary form as

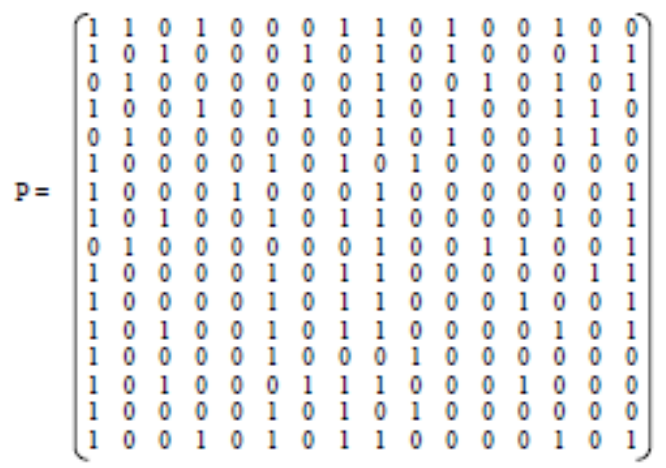

Let us choose a key $\mathrm{k}$ consisting of 32 decimal numbers. Thus, we have

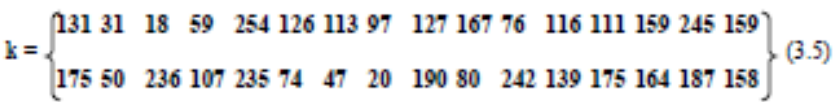

Then $\mathrm{k}$ can be written in the form of a matrix given by

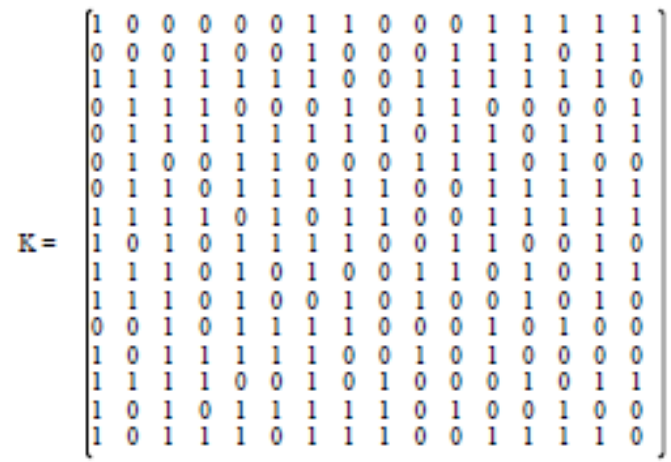

On using the algorithm given in section 2, the modular arithmetic inverse of $\mathrm{K}$ can be obtained as

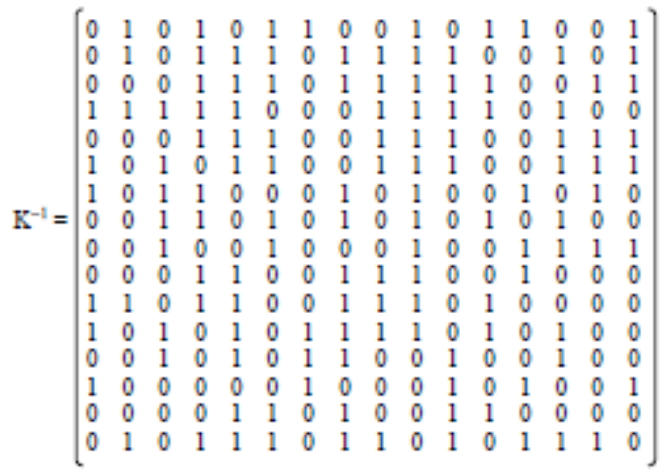

On using (3.6) and (3.7), it can be readily shown that $K K^{-1} \bmod 2=K^{-1} K \bmod 2=I$.

On applying the encryption algorithm, described in Section 2, we get the cipher text $\mathrm{C}$ in the form 


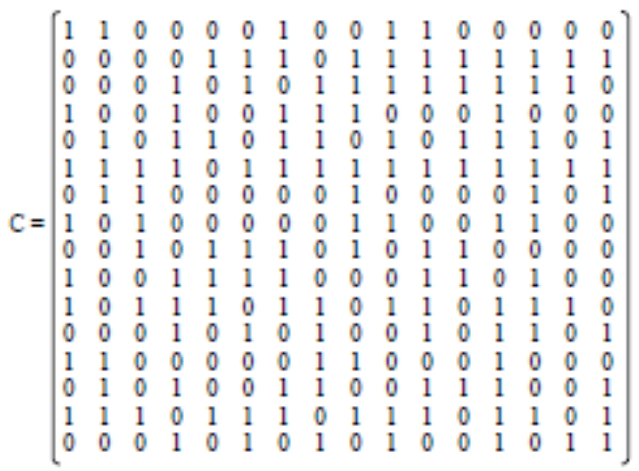

On using (3.6), (3.7), and (3.9), and applying the decryption algorithm described in section 2, we get the Plain text $\mathrm{P}$, which is the same as (3.4).

Let us now discuss the avalanche effect. Here, we modify the $11^{\text {th }}$ character ' $e$ ' in (3.2) to ' $d$ '. Then the plain text changes only in one binary bit as the EBCDIC codes of e and $\mathrm{d}$ are 85 and 84 respectively.

On using the modified plain text and the encryption algorithm, we get the cipher text $\mathrm{C}$ in the form

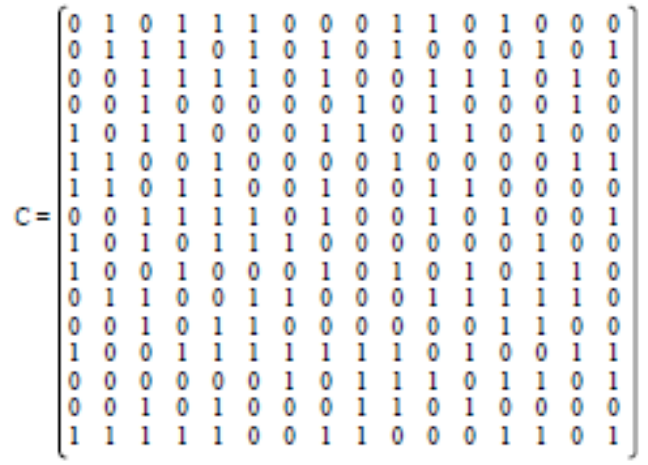

On comparing (3.9) and (3.10), we find that the two cipher texts differ in 132 bits out of 256 bits, which is quite significant.

Now let us change the key in (3.5) by 1 binary bit. This can be achieved by replacing the $3^{\text {rd }}$ element 18 of the key k by 19 . Then on using the original plain text (3.4), the modified key and the encryption algorithm, we get $\mathrm{C}$ in the form

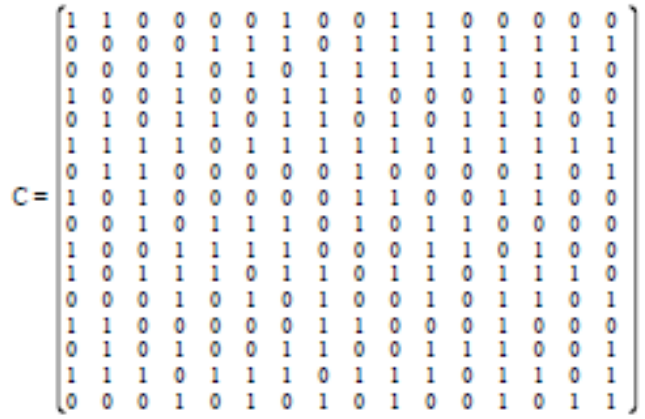

(3.11)

On comparing (3.11) with (3.9), we find that the cipher texts differ in 114 bits.

From the above analysis, we find that the Avalanche effect is quite pronounced and hence the cipher is a strong one.

By decomposing the entire plain text given by (3.1) into blocks, wherein each block is of size 32 characters, the corresponding cipher text can be written in hexadecimal notation in the form

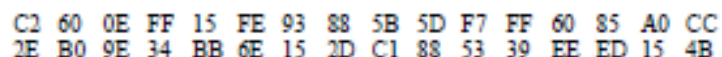
$\begin{array}{lllllllllllllllll}A 4 & 2 A & 12 & 36 & 93 & 3 F & 56 & 53 & 3 B & 7 E & 00 & 9 C & 60 & 3 B & O E & D 8\end{array}$ $\begin{array}{lllllllllllllllll}26 & 82 & 73 & 60 & \text { E7 } & \text { FB } & 49 & 2 C & \text { E1 } & 52 & \text { CC } 20 & 28 & 2 \mathrm{D} & \text { EA } & 65\end{array}$

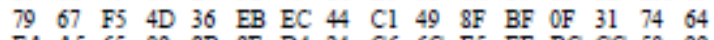
EA A5 65 09 $8 \mathrm{~B}$ 9E $\mathrm{D} 424$ C6 $6 \mathrm{C}$ E5 $\mathrm{EE}$ BC $C \mathrm{CC} 5099$

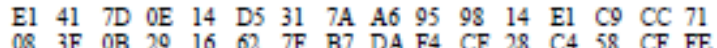

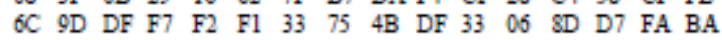
$\begin{array}{llllllllllllllll}3 \mathrm{~B} & 94 & \mathrm{~F} 6 & 01 & 8 \mathrm{~F} & 9 \mathrm{C} & 2 \mathrm{C} & 2 \mathrm{~A} & \mathrm{EC} & 79 & \mathrm{~A} 4 & 2 \mathrm{~F} & 56 & \mathrm{DD} & 99 & 7 \mathrm{E} \\ \mathrm{A} 3 & 63 & \mathrm{D} 9 & \mathrm{~B} 8 & \mathrm{AA} & \mathrm{OC} & 8 \mathrm{D} & 07 & \mathrm{CC} & 38 & 0 \mathrm{C} & 4 \mathrm{~F} & \mathrm{EC} & 27 & \mathrm{Bg} & 8 \mathrm{C}\end{array}$ 03 FF $\mathrm{FA}$ CB 69 90 A0 DD $62 \quad 55 \quad 39$ F5 $\mathrm{BF} \quad \mathrm{AD} 66 \quad \mathrm{ED}$ $\begin{array}{llllllllllllllll}76 & 3 \mathrm{~B} & \mathrm{EF} & \mathrm{A} 2 & 7 \mathrm{E} & 5 \mathrm{E} & 29 & 78 & 7 \mathrm{~B} & \mathrm{FC} & \mathrm{DE} & \mathrm{DA} & 82 & 64 & \mathrm{BE} & \mathrm{C} 3\end{array}$

$\begin{array}{llllllllllllllllllllll}49 & \mathrm{D} 9 & 4 \mathrm{~B} & \mathrm{CB} & 9 \mathrm{~F} & 71 & \mathrm{FC} & 5 \mathrm{E} & 86 & 8 \mathrm{~B} & 04 & 2 \mathrm{~B} & 31 & 48 & \mathrm{E} 8 & 3 \mathrm{C} & (3.12)\end{array}$

The problem under consideration can also be studied, in the case when $\mathrm{K}$ and $\mathrm{K}^{-1}$ are interchanged, i.e., when (2.4) is replaced by

$\mathbf{P}=\left(\mathbf{K}^{-1} \mathbf{P} \mathbf{K}\right) \bmod 2$.

This amounts to interchanging $\mathrm{K}$ and $\mathrm{K}^{-1}$ in (2.4). Corresponding changes can be made in the algorithms. In this case, the $\mathrm{C}$ is given by

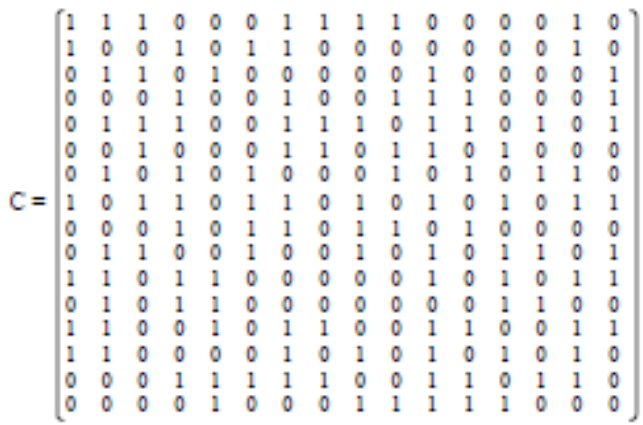

Though we have got a different cipher text, on account of modifications, we have obtained the same plain text $\mathrm{P}$ by performing decryption.

When the plain text $\mathrm{P}$ is changed by one bit (i.e., the $11^{\text {th }}$ character ' $\mathrm{e}$ ' is changed to ' $\mathrm{d}$ '), then the corresponding cipher text assumes the form

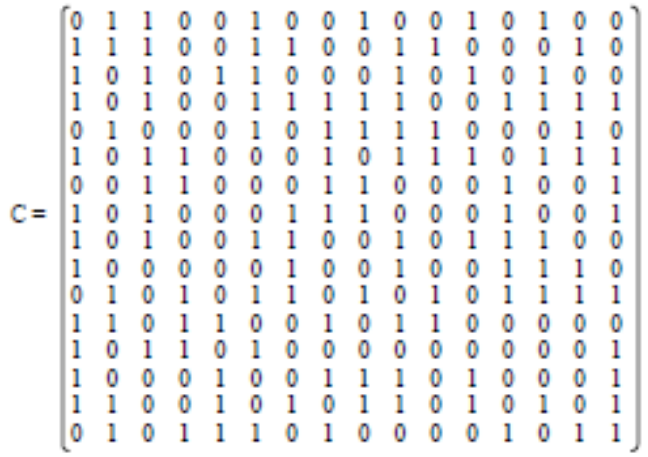

(3.15)

Thus in this case, the change in the cipher text is 133 bits out of 256 bits.

On changing 1 bit in the key (i.e., replacing 18 by 19), we have

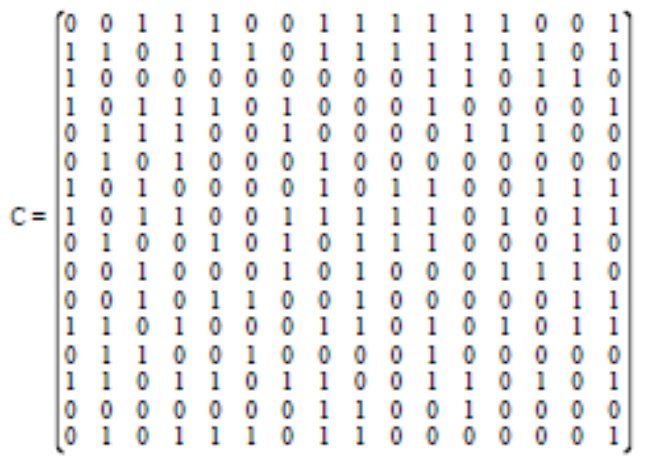


From (3.14) and (3.16), we notice the change in C is 123 bits out of 256 bits. From the above analysis, we find that the Avalanche effect is quite significant and hence this cipher is also is a very strong one.

In this case, the cipher text corresponding to the entire plain text (3.1), in hexadecimal form, is given by

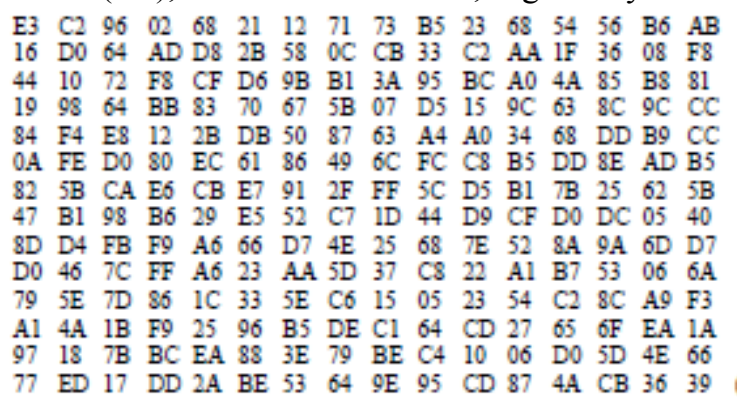

\section{CRYPTANALYSIS}

The different types of cryptanalytical attacks available in the literature are:

(1) Cipher text only attack,

(2) Known plain text attack,

(3) Chosen plain text attack,

(4) Chosen cipher text attack.

When the cipher text is known to us, we can determine the plain text, if the key is known. As the key contains 32 decimal numbers, the key space is of size

$$
2^{256} \simeq\left(10^{3}\right)^{25.6}=10^{76.8} \text {. }
$$

As the computation of the cipher text corresponding to all possible keys would take a very large amount of time, the cipher cannot be broken by the brute force approach.

We know that, the Hill cipher can be broken by the known plain text attack, as we can form a direct relation between $\mathrm{C}$ and $\mathrm{P}$. But in the present modification, which involves $\mathrm{K}$ and $\mathrm{K}^{-1}$, one on the left side of $\mathrm{P}$ and the other on the right side of $\mathrm{P}$, and the process of iteration together with the Mix function and the XOR operation, we cannot get a direct relation between $\mathrm{C}$ and $\mathrm{P}$. Hence, this cipher developed in this analysis cannot be broken by the known plain text attack.

The chosen plain / cipher text attack is totally ruled out as the steps involved in the cipher are typical in nature.

\section{CONCLUSIONS}

In this paper, we have modified the Hill cipher, governed by the single relation

$$
\mathrm{C}=(\mathrm{K} P) \bmod 26 \text {, }
$$

in two different cases.

In case one, the iterative scheme includes the relations

$$
\begin{aligned}
& \mathbf{P}=\left(\mathbf{K ~ P ~ K}^{-1}\right) \bmod 2, \\
& \mathbf{P}=\operatorname{Mix}(\mathbf{P}), \\
& \text { and } \mathbf{P}=\mathbf{P} \oplus \mathbf{K},
\end{aligned}
$$

(5.4)

and in case two, it includes the relations

$$
\begin{aligned}
& \mathbf{P}=\left(K^{-1} \mathbf{P} \mathbf{K}\right) \bmod 2, \\
& \mathbf{P}=\operatorname{Mix}(\mathbf{P}),
\end{aligned}
$$

and $\mathbf{P}=\mathbf{P} \oplus \mathbf{K}$.

In this analysis, the length of the plain text block is 256 bits and the length of the cipher text block is also 256 bits. As the cryptanalysis clearly indicates, this cipher is a strong one and it cannot be broken by any cryptanalytic attack. This analysis can be extended to a block of any size by using the concept of interlacing [2].

\section{REFERENCES}

[1] V. U. K. Sastry, D. S. R. Murthy, S. Durga Bhavani, "A Block Cipher Involving a Key Applied on Both the Sides of the Plain Text", International Journal of Computer and Network Security (IJCNS), Vol. 1, No. 1, pp. $27-30$, Oct. 2009.

[2] V. U. K. Sastry, V. Janaki, “A Modified Hill Cipher with Multiple Keys", International Journal of Computational Science, Vol. 2, No. 6, 815 - 826, Dec. 2008.

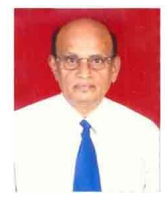

Dr. V. U. K. Sastry is presently working as Professor in the Dept. of Computer Science and Engineering (CSE), Director (SCSI), Dean (R \& D), SreeNidhi Institute of Science and Technology (SNIST), Hyderabad, India. He was Formerly Professor in IIT, Kharagpur, India and worked in IIT, Kharagpur during 1963 - 1998. He guided $12 \mathrm{PhDs}$, and published more than 40 research papers in various international journals. His research interests are Network Security \& Cryptography, Image Processing, Data Mining and Genetic Algorithms.

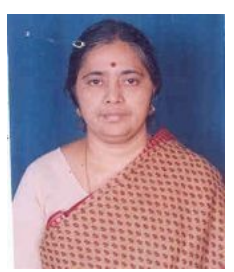

Dr. S. Durga Bhavani is presently working as Professor in School of Information Technology (SIT), JNTUH, Hyderabad, India. She has more than 18 years of teaching experience. Her research area includes Evidential Reasoning, Cryptography and Image Processing. She has no. of research publications to her credit.

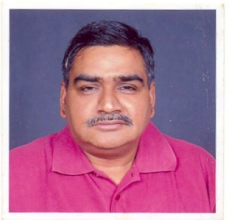

Prof. D. S. R. Murthy obtained B. E. (Electronics) from Bangalore University in 1982 M. Tech. (CSE) from Osmania University in 1985 and presently pursuing Ph.D. from JNTUH, Hyderabad since 2007 . He is presently working as Professor in the Dept. of Information Technology (IT), SNIST since Oct. 2004. He earlier worked as Lecturer in CSE, NIT (formerly REC), Warangal, India during Sep. 1985 - Feb. 1993, as Assistant Professor in CSE, JNTUCE, Anantapur, India during Feb. 1993 - May 1998, as Academic Coordinator, ISM, Icfaian Foundation, Hyderabad, India during May 1998 - May 2001 and as Associate Professor in CSE, SNIST during May 2001 - Sept. 2004. He worked as Head of the Dept. of CSE, JNTUCE, Anantapur during Jan. 1996 - Jan 1998, Dept. of IT, SNIST during Apr. 2005 - May 2006, and Oct. 2007 - Feb. 2009. He is a Fellow of IE(I), Fellow of IETE, Senior Life Member of CSI, Life Member of ISTE, Life Member of SSI, DOEACC Expert member, and Chartered Engineer (IE(I) \& IETE). He published a text book on C Programming \& Data Structures. His research interests are Image Processing and Image Cryptography and published research papers in International Journal of Computer and Network Security (IJCNS) and International Journal of Computer Theory and Engineering (IJCTE). 